NASA Contractor Report 182245

AIAA-89-0007

\title{
Comparison of 3D Computation and Experiment for Non- Axisymmetric Nozzles
}

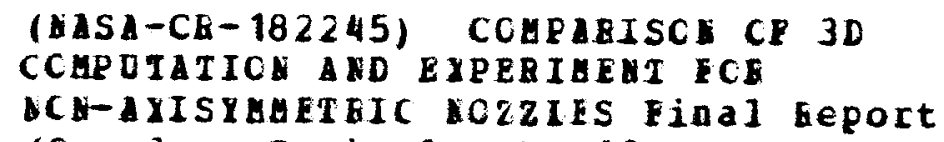

H. Lai and E. Nelson

Sverdrup Technology, Inc.

NASA Lewis Research Center Group

Cleveland, Ohio

February 1989

Prepared for

Lewis Research Center

Under Contracts NAS3-24105 and NAS3-25266

\section{N/Sก \\ National Aeronautics and \\ Space Administration}


COMPARISON OF 3D COMPUTATION AND EXPERIMENT FOR NON-AXISYMMETRIC NOZZLES

\author{
H. Lai ${ }^{*}$ and E. Nelson* \\ Sverdrup Technology, Inc. \\ NASA Lewis Research Center Group \\ Cleveland, Ohio 44135
}

\begin{abstract}
Three dimensional solutions of a single expansion ramp nozzle are computed with the existing PARC computer code by solving the full Navier-stokes equations. The computations are performed to simulate the non-axisymmetric nozzle flowfield in both the internal/external expansion regions and the exhaust plume in a quiescent ambient environment. Two different configurations of the nozzle at a pressure ratio $N P R=10$ are examined. Numerical results of laminar flows are presented, and the wall pressure distributions are compared with the experimental data.
\end{abstract}

\section{Introduction}

Numerical simulations of non-axisymmetric nozzles are described in the present paper. The configurations are selected from a set of the single expansion ramp nozzles which were experimentaliy investigated by $R e$ and Leavitt [1]. The experiments were performed to analyze the effects of various geometrical parameters and pressure ratios on the static performance of these asymmetric nozzles.

The present converging/diverging nozzle has a longer upper surface functioning as an external expansion ramp, with a rectangular cross-section in the internal nozzle region. The edge of the flat sidewall is highly skewed at the nozzle exit to connect the upper and lower surface lips. The flow generated by large streamwise pressure differences expands from near reservoir conditions through the nozzle and exhausts supersonically into the quiescent air. The resulting flowfield is characterized by flow expansion inside the nozzle and by the exhaust plume interaction with the ambient environment. In particular, a strongly interactive flow structure containing a normal shock, expansion/compression wave reflection, and separation can occur in the region along the external expansion ramp of the upper surface. Around this section, the free shear layer emerging from the lip of

* Research Engineer, Member AIAA the shorter lower surface acts as an artificial nozzle wall, and its trajectory shape can alter the nature of the flow, depending on the ambient conditions below it. The wave structure initiated by the shear layer possibly includes an oblique shock, or an expansion fan emanating from the lower nozzle lip. This shear layer itself can also deflect upwards at a high angle to interact with the upper boundary layer. These characteristics of the flow are three-dimensional. A similar pattern exists in the spanwise direction where the sidewall creates a vertical free shear surface starting at the skewed nozzle exit. Furthermore, at high Reynolds number, the shear layer behind a sharp or blunt trailing edge may not be stable. High Reynolds number interaction of the free shear layer is even more unstable in a quiescent external environment than in a subsonic or supersonic external stream. This behavior has been observed by the present authors and others [13] in numerical computations. consequently, a steady state solution may not possible for certain flows with a quiescent external stream. The threedimensionality of the flowfield, its unsteadiness, and stability considerations are some of the complications of the present problem, although only steady-state solutions are computed in this study.

Applications of numerical methods to the nozzle complex flowfield above have been conducted in many studies. Some of these studies have focused on the interaction of the exhaust plume and the external stream, and the consequent effects on the nozzle afterbody $[2,3]$. In several other studies, the jet plume has been analyzed independently $[4,5]$. The flow inside the nozzle is treated as a separate problem, and is generally well understood for a simple two-dimensional symmetrical nozzle. In the case of the nozzle considered in this paper, however, the independent treatment of the exhaust plume may not be adequate, since the initial part of the exhaust plume bordered by its free shear layer can change the flow structure near the upper external expansion surface as discussed previously. Simultaneously solving the flowfield to account for the strongly interactive nature thus becomes necessary. Early and recent works related to this type of nozzle, in 
which the computations include a complete domain of the internal nozzle, the external exhaust plume and the ambient stream, have been performed by several other investigators [6-9]. Independent calculation of a similar nozzle has also been reported recently for a two-dimensional case [10]. other results based on the full Navierstokes (NS) equations, parabolized NavierStokes (PNS) equations or method of charateristics in two and three dimensions have been calculated for a variety of nozzle conditions including supersonic, subsonic and quiescent external streams. In addition, these previous analyses have demonstrated the simplicity of the simulation procedures as applied to the nozzle/exhaust problem, in which the geometry is complex and the flow is highly nonuniform.

In the present paper, the existing PARC code is employed to compute the solutions of the complete nozzle/exhaust flowfield. The PARC code solves the full Navier-stokes equations written in a 3D generalized curvilinear coordinate system. The detailed development and some of the recent work related to this computer program can be found in refs. [1115]. The numerical formulation in the PARC code will be summarized in the following section.

Irregular geometries with embedded surfaces can be implemented in a simplified manner with the PARC code. A convenient $\mathrm{H}$-grid is obtained using simple algebraic grid generation. Two configurations of the asymmetric, single expansion ramp nozzle are simulated at a pressure ratio (jet stagnation pressure to ambient static pressure) $\mathrm{NPR}=10$. At this pressure ratio, the flow is predominantly inviscid, and the viscous effect is only important along the wall boundary layer and within the stable shear layer. The assumption of laminar flow hence can be applied appropriately. The converged solutions are compared with the experimental measurements for the pressure distributions on the upper and lower nozzle surfaces.

\section{Solution Method}

Steady-state solutions of the transformed compressible Navier-stokes equations are obtained from the PARC code by a timemarching finite-difference scheme. The numerical scheme employs three-point central differences uniformly throughout the flowfield to approximate the spatial derivatives. The time term is implicitly discretized by a two-point backward formula. For numerical stability, second- and fourthorder Jameson-type artificial dissipation is included. This artificial damping addition leads to a block pentadiagonal system of equations. Further simplification to a scalar pentadiagonal system has also been implemented in the existing PARC code using the diagonalization of the inviscid terms.
This efficient diagonalized formulation reduces the computational effort significantly as compared to the original approach. The resulting time-linearized discrete equations in the delta form are solved by the ADI Beam and Warming approximate factorization. Each ADI step requires inversions of scalar pentadiagonal systems. The implicitness of the algorithm refers to the coupling of the inviscid terms, whereas the viscous diffusion part is treated explicitly at the previous time step. A limitation of the temporal step size, therefore, arises when computing viscous flows. Since very small spatial resolution is required in the viscous regions, a rather small time step must be used, and consequently the convergence rate is slow. A variable local time step based on an approximate linearized CFL condition is used to alleviate this restriction so that larger time steps can be taken in regions with coarse resolution. starting from an assumed initial flowfield, the final solution is obtained by marching the governing equations with the numerical scheme in time to steady state or convergence.

All boundary conditions are treated explicitly. In the present nozzle, there is a plane of symmetry in the spanwise (z) direction. Only half of the nozzle then needs to be computed. Symmetry conditions are specified on this center plane. No-slip velocity and adiabatic wall temperature are imposed on the nozzle surfaces. In the farfield, still air conditions, at a pressure of $101.3 \mathrm{kPa}$ and a temperature of $300 \mathrm{~K}$, are fixed at the upper and lower boundaries in the vertical (y) direction at a sufficiently large distance from the nozzle. Similar boundary conditions are imposed at the farfield in the spanwise direction. In the streamwise ( $x$ ) direction, a stagnation pressure of $1013 \mathrm{kPa}$ and a stagnation temperature of $300 \mathrm{~K}$ are specified at the nozzle entrance. Other variables at this location are computed using the isentropic relationships and a characteristic-like condition extrapolated from the interior. At the outflow boundary, streamwise flux gradients are assumed negligible.

For normalization, stagnation quantities at the nozzle entrance are taken as the reference variables. The nozzle throat height and the speed of sound are the reference length and velocity, respectively. The Reynolds number is computed based on these values. The reference temperature, pressure and length are taken to be $300 \mathrm{~K}$, $1013 \mathrm{kPa}$, and $2.54 \mathrm{~cm}$, respectively. The corresponding Reynolds number is 5.618 million. Laminar viscosity is obtained from the sutherland law, and the Prandtl number is assumed to be equal to 0.72 . 
Numerical Results

Geometry:

The geometry of the single expansion ramp nozzle was obtained from ref. [1], in which a detailed experimental study has been documented for several geometrical parameters and pressure ratios. The general configuration of the nozzle used in the experiment is reproduced here in fig. (1). The basic components of the nozzle consist of an upper two-dimensional flap and a shorter lower two-dimensional flap. These flaps are flat surfaces in the spanwise direction. A section of the upper flap extending from the throat area functions as an external expansion ramp. The vertical sidewall is shown in this figure and, as indicated, the edge of the sidewall is highly inclined at a large angle with respect to the vertical direction. This arrangement of the components gives a rectangular cross-section parallel to the exit plane aligned with the tilted edge. Intersections of the tilted edge and the upper and lower nozzle walls normally occur upstream of the ends of the wall surfaces.

Two nozzle configurations are examined numerically. The geometrical difference between the two cases is depicted in $\mathrm{fig}$. (2), showing the contour shapes of the upper and lower walls. Both nozzles have identical lower flaps and sidewalls. It is noted that the flow expands to a lower pressure in case 2 , because the external expansion ramp section is longer than that of the first configuration. In both cases, the nozzle aspect ratio of width to height is 4 , and the height is measured at the throat section. The flat sidewall is assumed to have a very thin thickness of $0.0058 \mathrm{~cm}$. Case 1 and 2 are refered to as case IT5 and OT5 respectively in ref. [1].

Grid:

The simulated flow includes the interior and exterior regions of the nozzle, containing the downstream exhaust region. Since the nozzle has a symmetrical plane at the middle in the spanwise direction, as mentioned above, the spanwise regions thus include the interior half of the nozzle and the flow exterior to the sidewall. The complete domain is then divided correspondingly into sub-sections to simplify grid generation using an algebraic technique. For resolution, hyperbolic tangent functions are used to cluster grid points in regions near the walls. Patching these separate segments produces a final sheared grid in Cartesian coordinates. The PARC code internally computes the metrics and Jacobian of the curvilinear coordinate transformation.

Figure (3a) illustrates a view of the nonuniform grid in the $x-y$ plane which is along the streamwise direction. The grid clustering concentrates near the wall surfaces and extends to the exhaust region behind the inclined nozzle exit. Fig. (3b) presents a detailed view of the same grid showing a high degree of grid nonorthogonality, particularly in the regions next to the exit. The skewed grid lines result since one of the vertical curvilinear coordinates is made to align with the sidewall tilted edge. This is done in order to simplify the application of the boundary conditions. Spanwise views of the grid distribution in the $y-z$ plane are shown at two different axial locations inside the nozzle section, fig. (4a) with the nozzle walls indicated, and downstream in the exhaust plume area, fig. (4b). The clustering of the vertical coordinates is about the interior and exterior surfaces of the sidewall, and these spanwise grids are not on a plane with constant $x$-coordinate.

The flow starts from the nozzle entrance, $\mathrm{x}=0$, where a high total pressure is prescribed. It expands through the converging/diverging and external ramp sections and exhausts into the downstream low pressure region. This low pressure region is bounded by the quiescent boundaries located at large distances from the nozzle. The farfield computational boundaries are approximated at distances of 25,10 and 8 characteristic lenghts in the $x, y$ and $z$ directions, respectively. The solutions discussed below have been computed on the same grid of $135 \times 120 \times 50$ for both configurations. Of these, $60 \times 40 \times 30$ grid points are employed in the interior side of the nozzle. The smallest grid size is on the order of 0.001 near the wall regions for the $y$ and $z$ directions. This grid size then gives typically two to four subsonic points in the dominant supersonic viscous layers. For the $x$ direction, the grid is slightly refined near the nozzle exit and is on the order of 0.01 . In the figures discussed below, $i$ and $l$ indicate the streamwise and spanwise grid indices respectively, while $x$ and $z$ represent the corresponding nondimensional coordinates.

Case 1:

The converged result of the first configuration is presented with the Mach number, density and pressure contours at several streamwise and spanwise crosssections. The contours are in equally spaced increments over the entire range of values unless otherwise noted. The Mach contours on the center plane in fig. (5a) show a typical exhaust plume containing a steady-state multiple inviscid cell pattern in the supersonic exhaust core. The flow is sonic at the throat and highly supersonic at the nozzle exit. The first inviscid cell appears very irregular and is bounded by an expansion/compression wave system. Because of the inclined trajectory of the exhaust plume, as can be seen, the Mach waves emanate from the ends of the flap surfaces, where the upper and lower free shear layers become the expansion and compression ramps. These shear layers depart from the nozzle walls at different angles. The lower shear 
layer is very diffusive and largely due to lack of grid resolution along this shear layer. The first cell has an average Mach number of 2.7. In the next two cells, the Mach numbers near the cell centers are about 2.3 and 2.0 respectively, showing a gradual decay of the core center velocities. The core cells reduce in size and numbers towards the sidewall as seen in figs. (5bd). The effects of the sidewall become pronounced in fig. (5d), with the appearance of the initial vertical shear layer. The flow inside of the nozzle has very thin viscous layers next to the walls, particularly on the lower surface, and thus appears predominantly inviscid. The spanwise contour views are shown in figs. (6a-d). Fig. (6a) presents the Mach contours at an axial station in the nozzle area, where the clustered regions indicate the upper, lower and sidewall surfaces. The contours in fig. (6b) are located on the external expansion ramp. The structure includes the lower shear layer, the thin vertical shear layer, and the upper boundary layer. In the exhaust region, these free shear layers become thicker with downstream distance, as shown in figs. $(6 c-d)$ in which the plume boundaries are evident. Figs. (7a-b) illustrate the density contours at the center plane and on the plane next to the sidewall, showing a pattern very much like Mach contours but out of phase. Additionally, density variation is small across both shear layers. This same variation also occurs for the pressure as seen in fig. (8), and the pressure decays quickly to the ambient value. The contours in Figs. $(7 a, 8)$ show a very weak wave system that is present in the exhaust core, particularly after the first inviscid cell. Figs. $(9 a-b, 10)$ depict typical density and pressure contours in the spanwise direction. These contours are plotted with smaller increments for clarity. It is noted that large variations in density and pressure take place inside the nozzle, and the exhaust flow does not deviate highly from the perfectly expanded condition. Finally, comparison is made with the measured data and presented in figs. (11a-b) for the upper and lower surfaces in the center plane. The agreement is very good for wall pressure distributions on both surfaces. This solution has been converged to five orders of magnitude in about 3000 time steps.

Case 2:

Solution of the second configuration is presented similarly in terms of the contours of the flow variables. Figs. (12a-d) depict the basic flow pattern of the supersonic exhaust into quiescent air. For this geometry, the upper plume boundary is welldefined by the very thin free shear layer which follows the upper nozzle contour, and remains nearly at this height downstream. The other shear layer is also deflected downwards and diffusive. Although the flow expands to about the same maximum Mach number indicated in case 1 , the supersonic inviscid core shown in fig. (12a) is more extensive. As a result, the fluctuating decrease of the core center velocities becomes smaller. Moreover, the flow is highly asymmetric in the region along the exhaust center line. The effect of the sidewall can be observed in fig. (12d) with the initial development of the vertical shear layer. It is noted that this shear layer is curved irregularly towards the center plane as it progresses downstream, since the spanwise velocity component is large and positive in the initial region of the vertical shear layer, but becomes small and negative downstream. The flow behind the inclined exit consists of two small separate supersonic regions next to the exit plane. spanwise views of the Mach contours at various axial locations are given in figs. $(13 a-d)$. The interior cross-sectional view is shown in fig. (13a) with a thicker expansion layer in the lower half of the nozzle. Figs. (13b-d) depict the contours at the axial plane on the external ramp region and the planes in the exhaust region. Density and pressure contours in the streamwise planes are shown in figs. (14a$b, 15)$. A very clear indication of the compression/expansion wave reflection can be observed at the first cell boundary in figs. $(14 \mathrm{a}, 15)$. The wave structure then becomes very weak with downstream distance. spanwise variations of density and pressure are illustrated in figs. $(16 a-b, 17)$. Comparison between the numerical prediction and experiment is presented in figs. (18a-b) with very good agreement for the wall pressure distributions in the center plane. The result has been obtained after approximately 3000 time steps with a residual reduction of three orders of magnitude. Apparently, case 1 gives a better residual reduction and a faster convergence rate by comparison although the flowfield is not markedly different from the second case. These two cases were computed with the same amount of artificial dissipation and the same maximum allowable time step.

\section{Summary}

Three-dimensional solutions obtained with the PARC code have been presented for two asymmetric, single expansion ramp nozzles at a pressure ratio of 10 . The computed flow consists of the internal expansion region in the converging/diverging sections and the external supersonic exhaust in a quiescent ambient environment. The fundamental characteristics existing at the prescribed flow condition have been captured successfully for the present nozzle/exhaust

flowfield. These features include expansion fans, Mach wave reflections, mixing layers, and non-symmetrical, multiple inviscid cell, supersonic exhausts. Comparison has been made with experimental data for wall pressure distributions at the center planes with good agreement. 
1. Re, R. J., and Leavitt, D. L., "Static Internal Performance of Single-ExpansionRamp Nozzles with Various Combinations of Internal Geometric Parameters," NASA TM 86270 , Dec 1984.

2. Putnam, L. E., and Hodges, J., "Assessment of NASA and RAE Vicous-Inviscid Interaction Methods for Predicting Transonic Flow over Nozzle Afterbodies," AIAA Paper No. 83-1789, July 1983.

3. Wilmoth, R., "Aerodynamics Interactions with Turbulent Jet Exhaust Plumes," Presented at the JANNAF 13th Plume Technology Meeting, April 1982, Houston, Texas.

4. Venkatapathy, E., and Feiereisen, W. J., "3-D Plume Flow Computations with an Upwind Solver," AIAA Paper No. 88-3158, AIAA/ASME/SAE/ASEE 24th Joint Propulsion Conference, July 1988, Boston, Ma.

5. Hasen, G. A., "Navier-Stokes Solutions for an Axisymmetric Nozzle," AIAA Journal, Vol. 20, sept. 1982, pp. 1219-1227.

6. Dash, S., and Del Guidice, P., "Shock Capturing Finite-Difference and Characteristic Reference Plane Techiques for the Prediction of Three-dimensional NozzleExhaust," NASA CR 145366, May 1978:

7. Thomas, P. D., "Numerical Method for Predicting Flow characteristiccs and Performance of Nonaxisymmetric Nozzles, Part 2 - Application," NASA CR 3264, Oct. 1980. 8. Peery, K. M., "Non-Axisymmetric Nozzle/Aftbody Flow Field Analysis," AFWALTR-81-3046, June 1981 .

9. Wolf, D. E., Lee, R. A., and Dash, S. M., "Parabolized Navier-stokes Analysis of Scramjet Hypersonic Nozzle Flowfields, "AIAA Paper No. 87-1897, AIAA/SAE/ASME/ASEE 23rd Joint Propulsion Conference, June 1987, San Diego, Ca.

10. Shieh, C. F., "Navier-Stokes Solutions of Transonic Nozzle Flow with Shock-Induced Flow Separations," AIAA Paper No. 88-3614, AIAA/ASME/SIAM/APS lst National Fluid Dynamics Congress, July 1988, Cincinnati, $\mathrm{OH}$.

11. Beam, R. M., and Warming, R. F., "An Implicit Factored Scheme for "the Compressible Navier-Stokes Equations," AIAA Journal, Vol.16, April 1978, pp. 393-402 12. Cooper, G. K., "The PARC Code: Theory and Usage," AEDC-TR-87-24, Oct. 1987.

13. Pulliam, T. H., "Euler and Thin Layer Navier-Stokes Codes: ARC2D, ARC3D," Notes for Computational Fluid Dynamics User's Workshop," The University of Tennessee Space Institute, Tullahoma, Tn., UTSI Pub. E024005-023-84, 1984, pp. 15.1-15.85.

14. Hsu, A. T., and Liou, M. S., "A Computational Analysis of Under-Expanded Jets in the Hypersonic regime," NASA TM $101319,1988$.

15. Harloff, G. J., Lai, H. T., and Nelson, E. S., "Two-Dimensional Viscous Flow Computations of Hypersonic scramjet Nozzle Flowfields at Design and off Design conditions," NASA TM 182150, June 1988.
This work was supported by the NASA Lewis Research Center under contracts NAS3-24105 and NAS3-25266 with Dr. Meng-Sing Liou as monitor. 
ORIGINAL PAGE IS

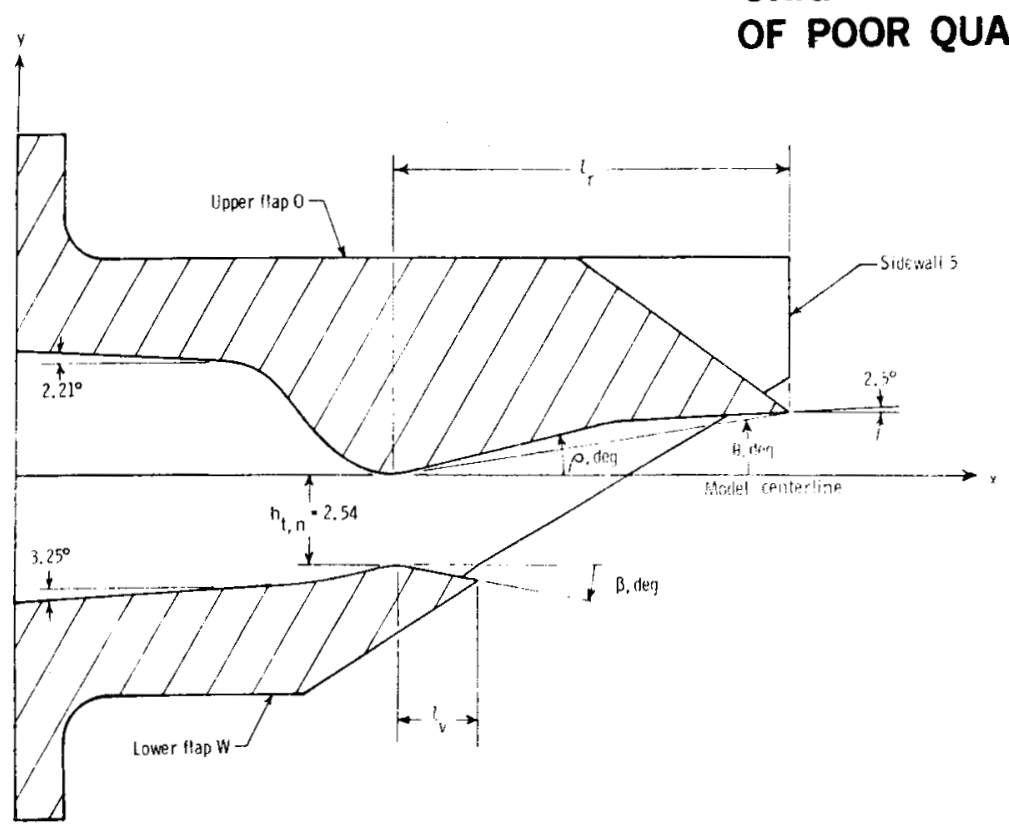

y

Fig. I Nozzle Geometry (From Ref. [1]).

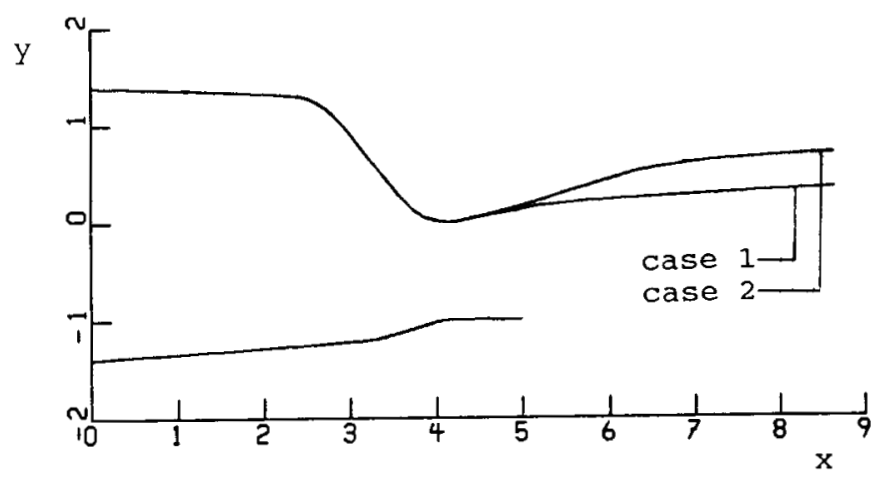

Fig. 4 Spanwise Grid Distribution:

a) In the Nozzle Region.

b) In the Exhaust Region.

Fig. 2 Comparison of Two Nozzle Configurations.

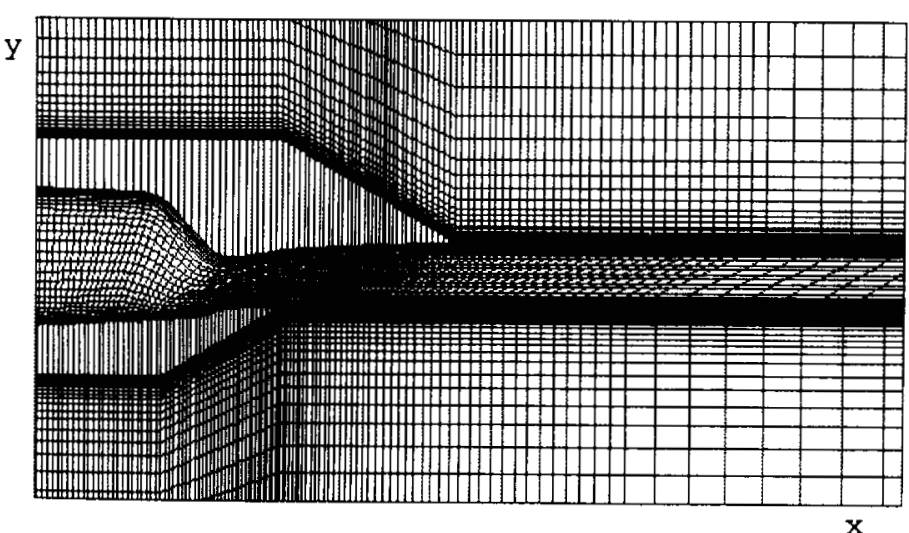

a)

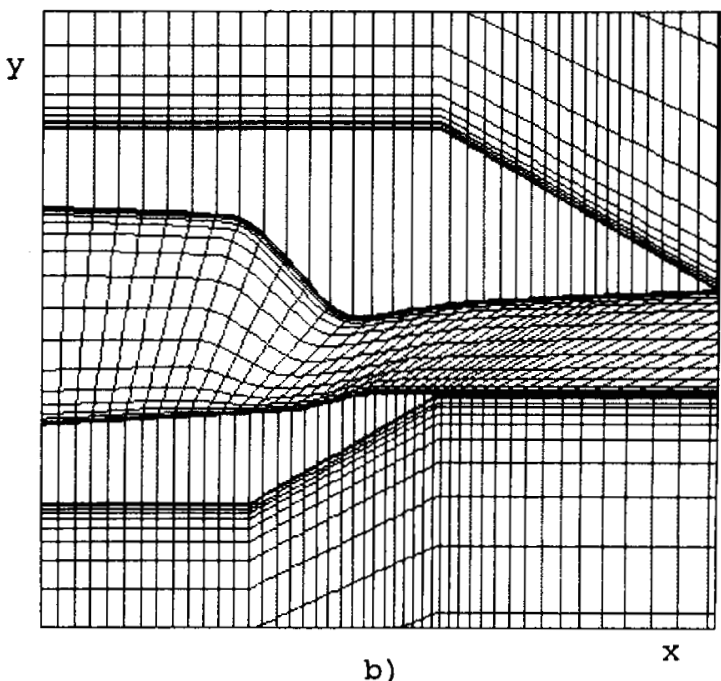

b)

Fig. 3 Streamwise Grid Distribution:

a) Nozzle and Exhaust gria.

b) Nozzle Grid. 


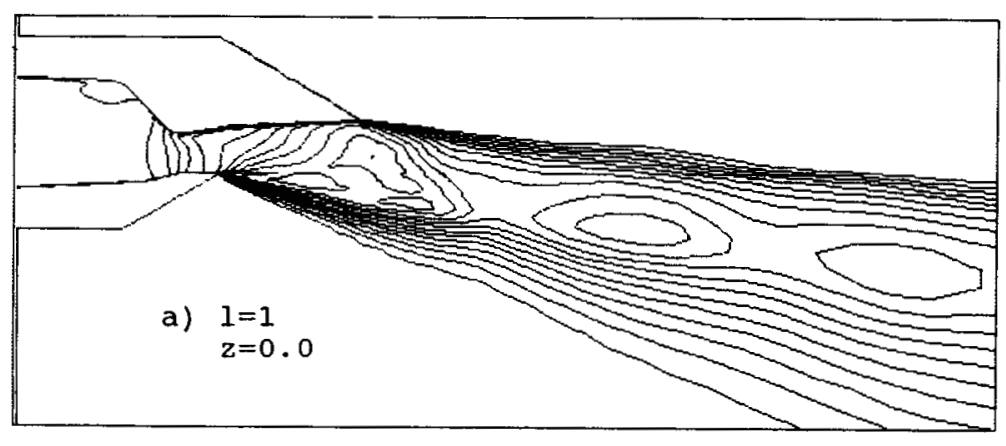

a)
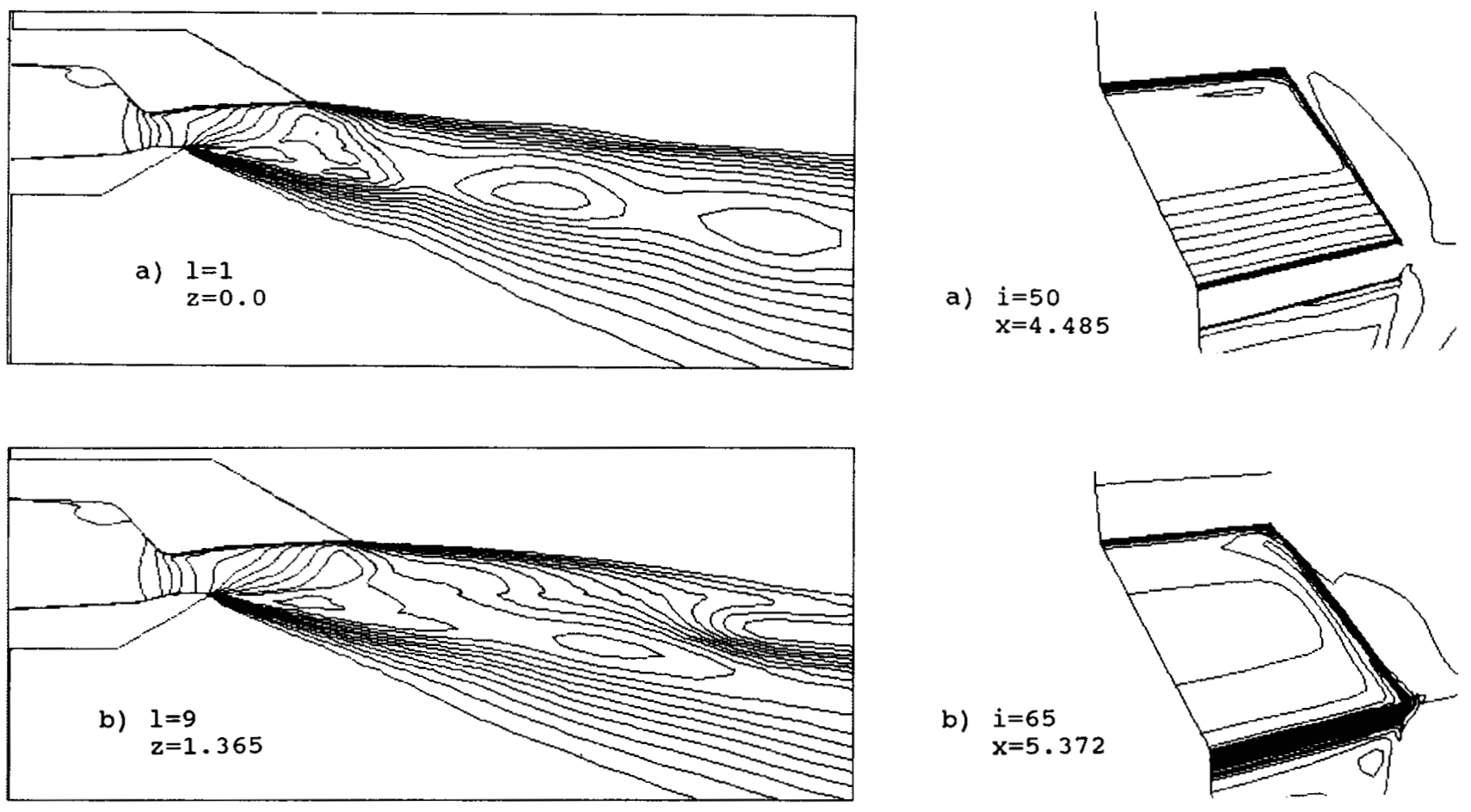

b) $i=65$

$x=5.372$
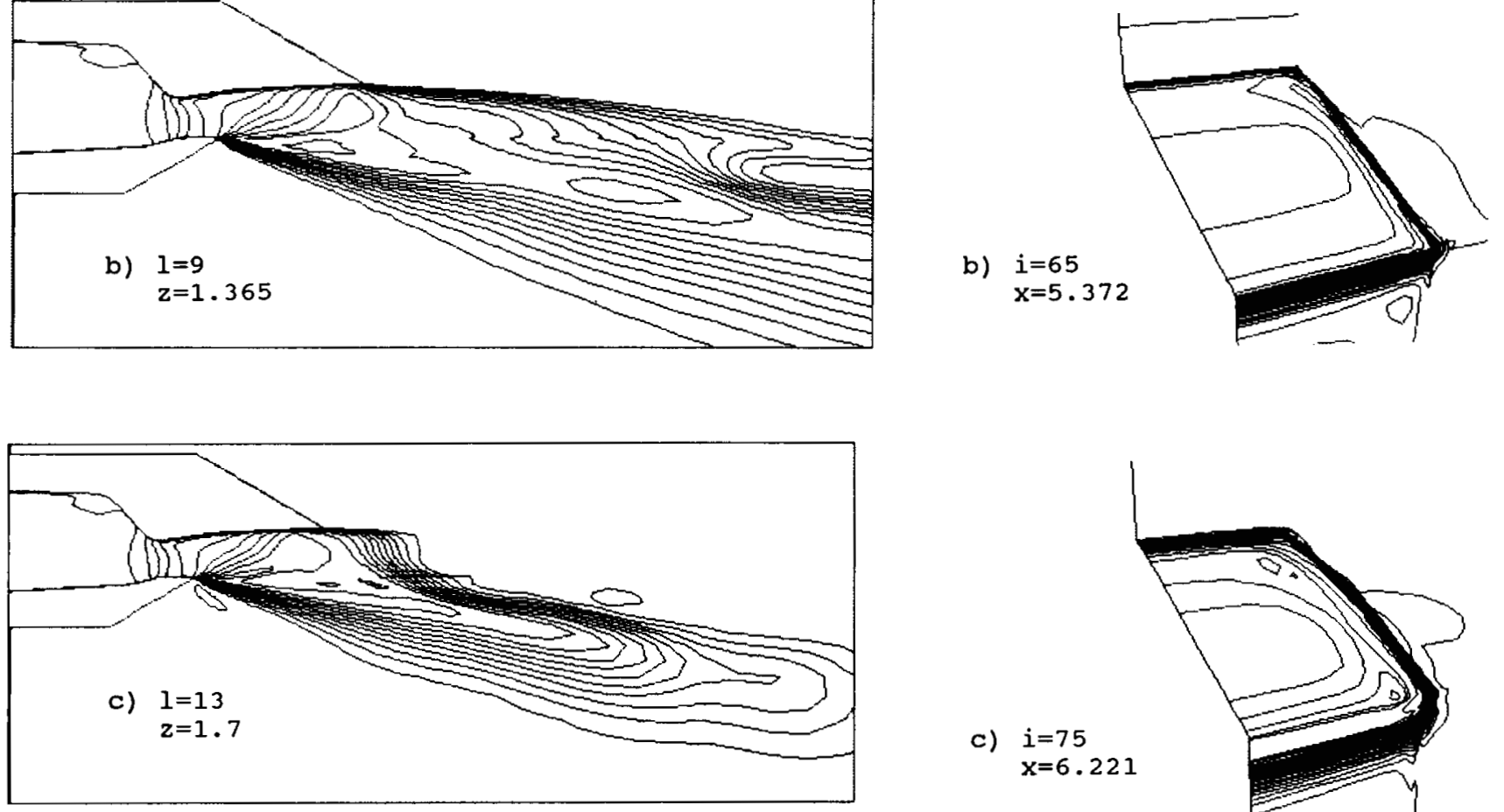

c) $i=75$

$x=6.221$
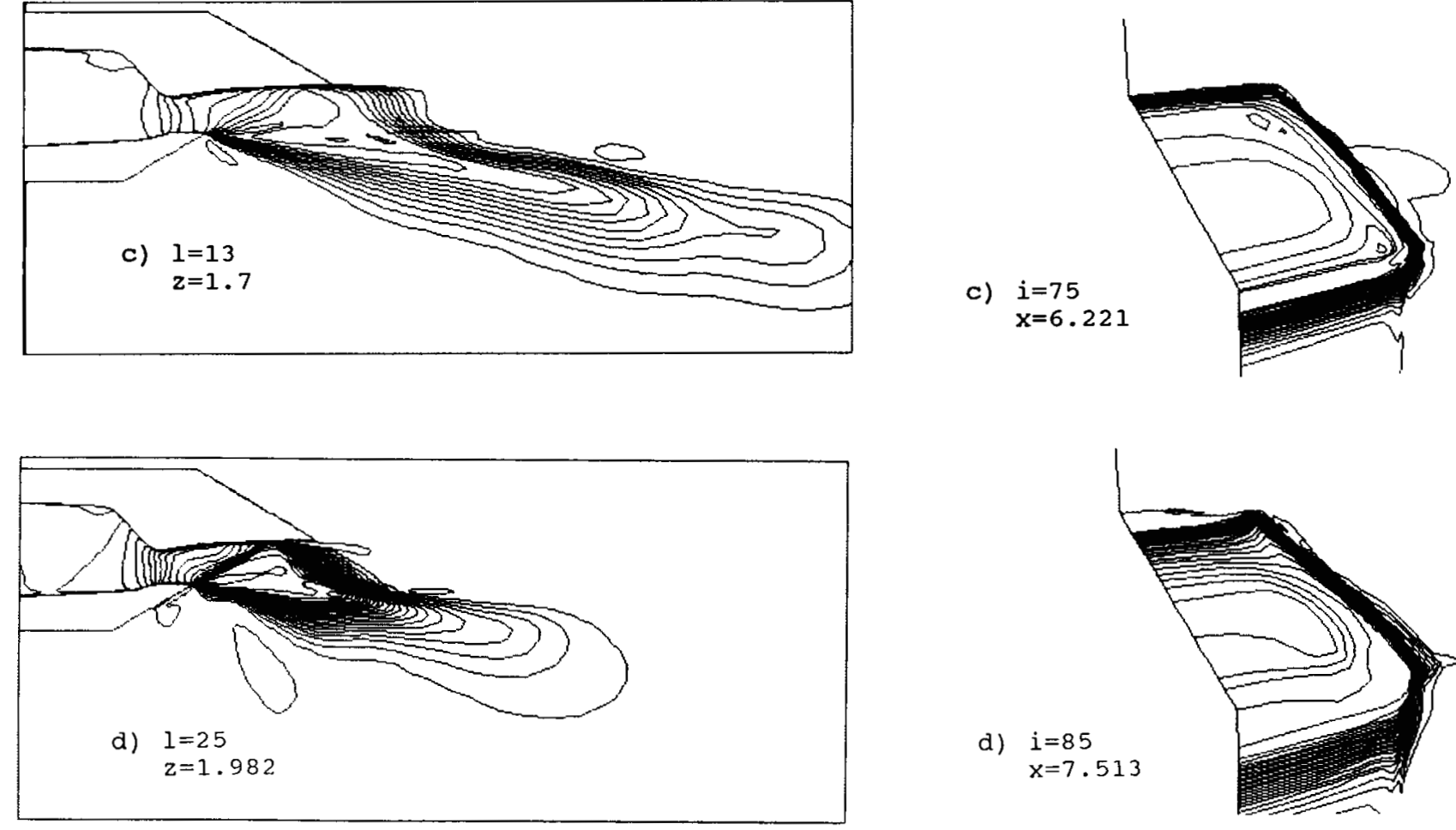

d) $\quad i=85$

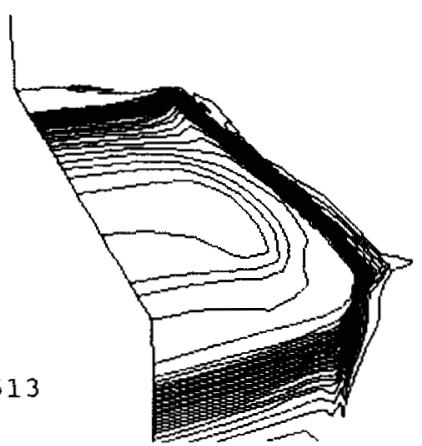

Fig. 5 Streamwise Mach Number Contours, Case 1.

Fig. 6 Spanwise Mach Number Contours, Case 1 . 


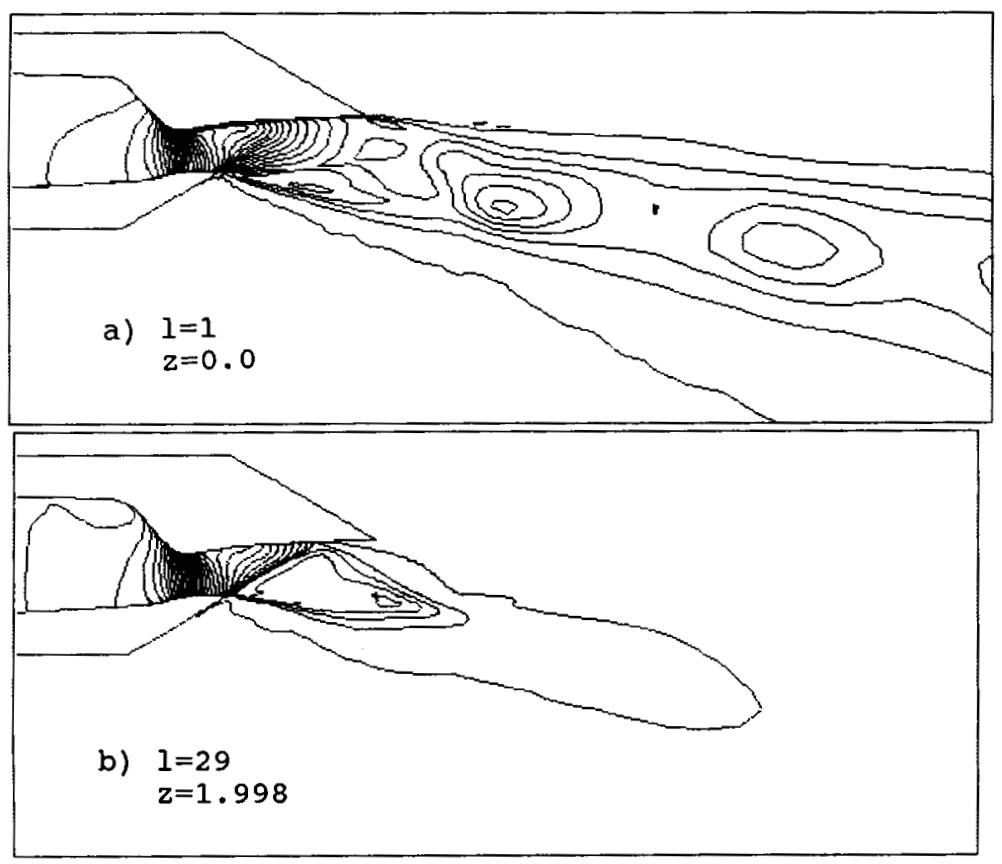

Fig. 7 Streamwise Density Contours, Case 1 .

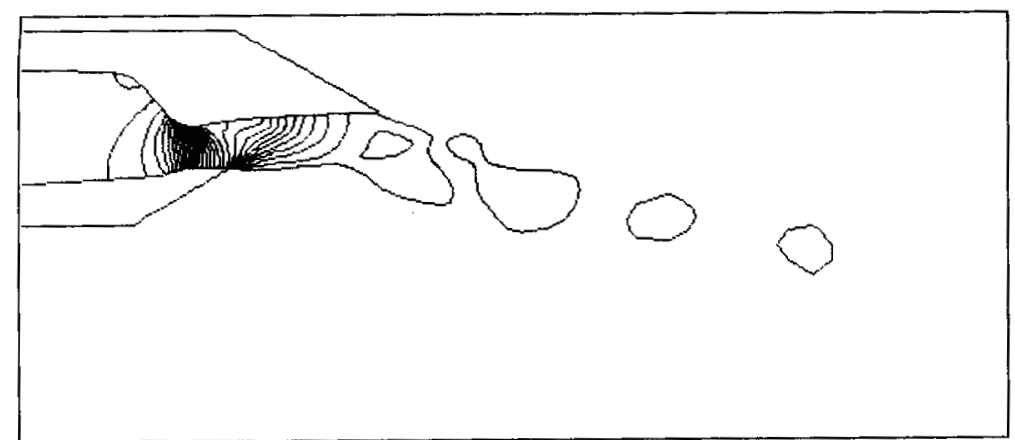

Fig. 8 Streamwise Pressure Contours, Case $1,1=1, x=0.0$.

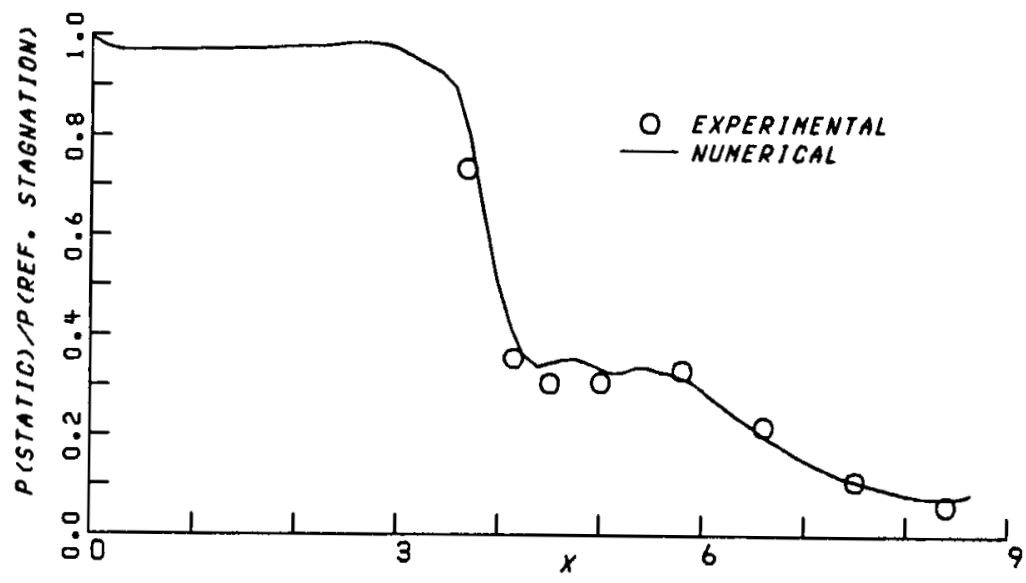

a) a) $i=85$

$x=7.513$

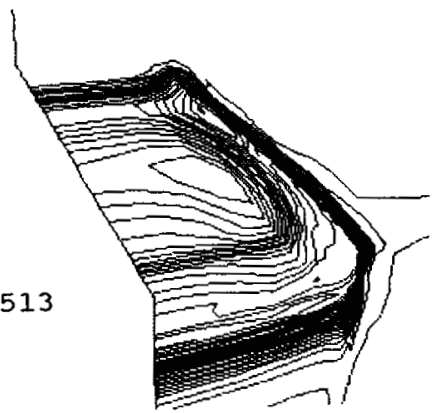

b) $i=95$

$x=9.419$

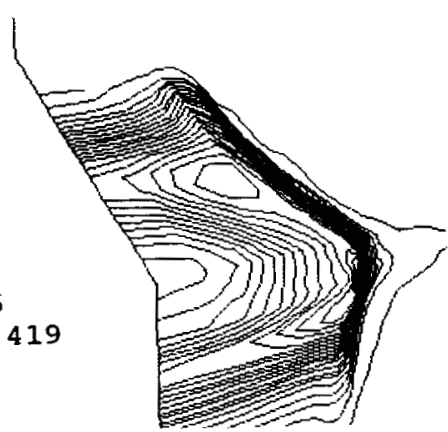

Fig. 9 Spanwise Density Contours, Case 1.

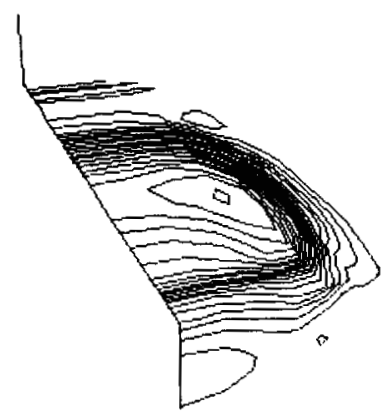

Fig. 10 Spanwise Pressure Contours, Case $1, i=85, x=7.513$.

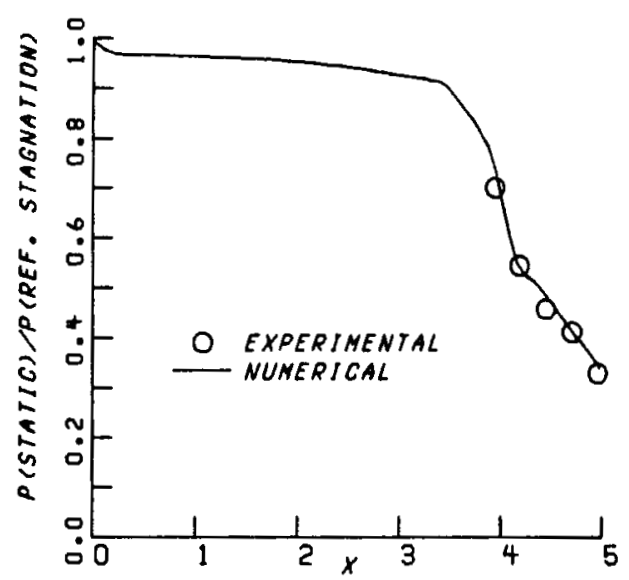

b)

Fig. 11 Wall Pressure Distributions, Case 1:

a) Upper wall

b) Lower wall 


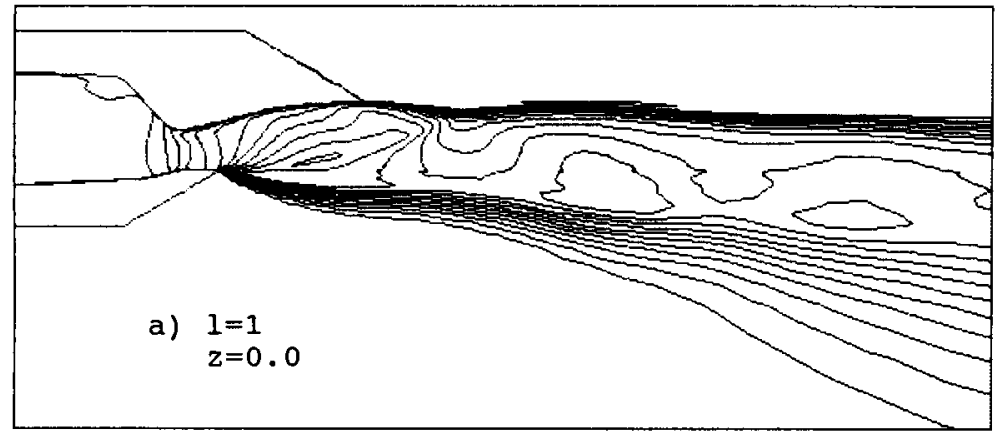

a) $i=53$

$x=4.673$
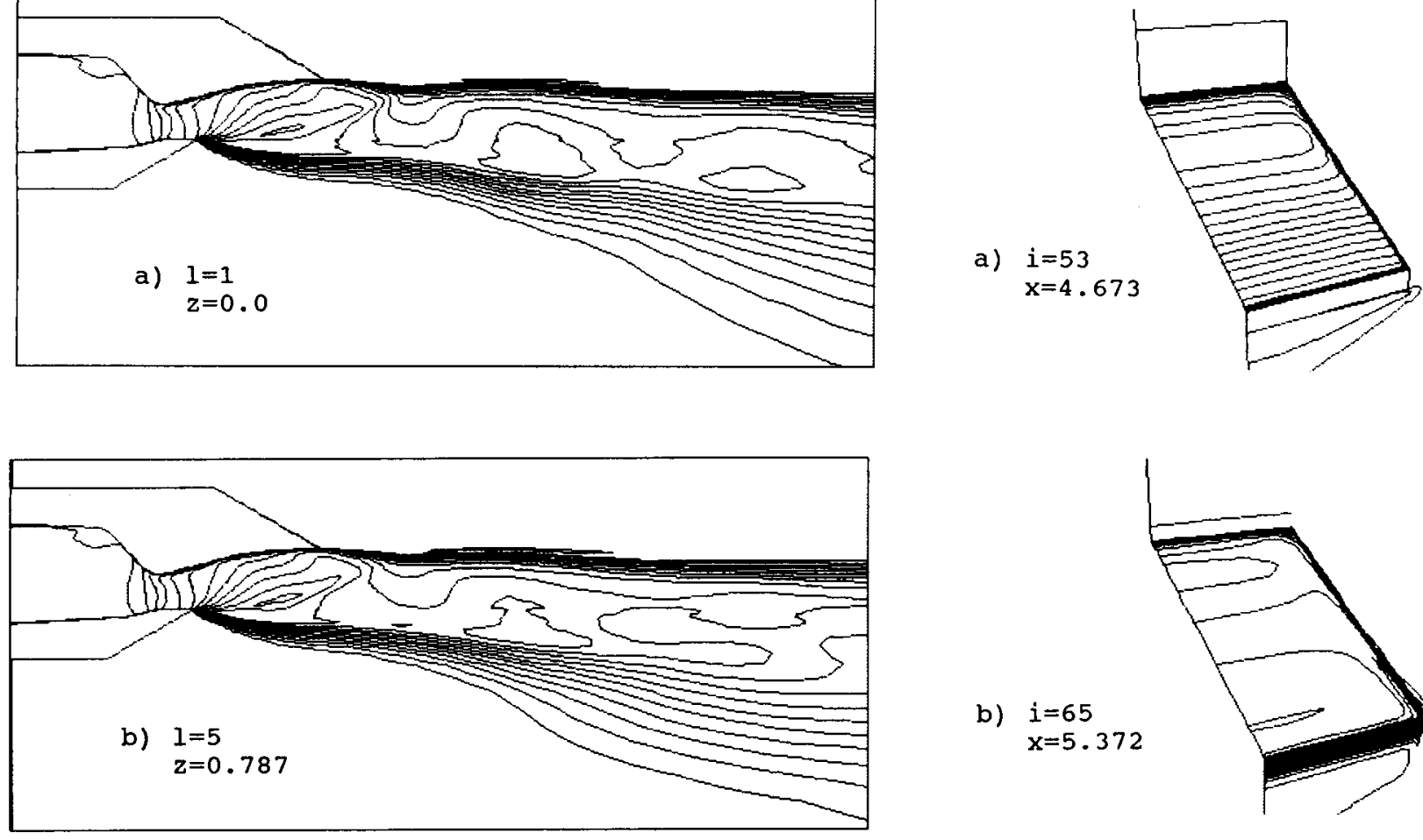

b) $i=65$

$x=5.372$
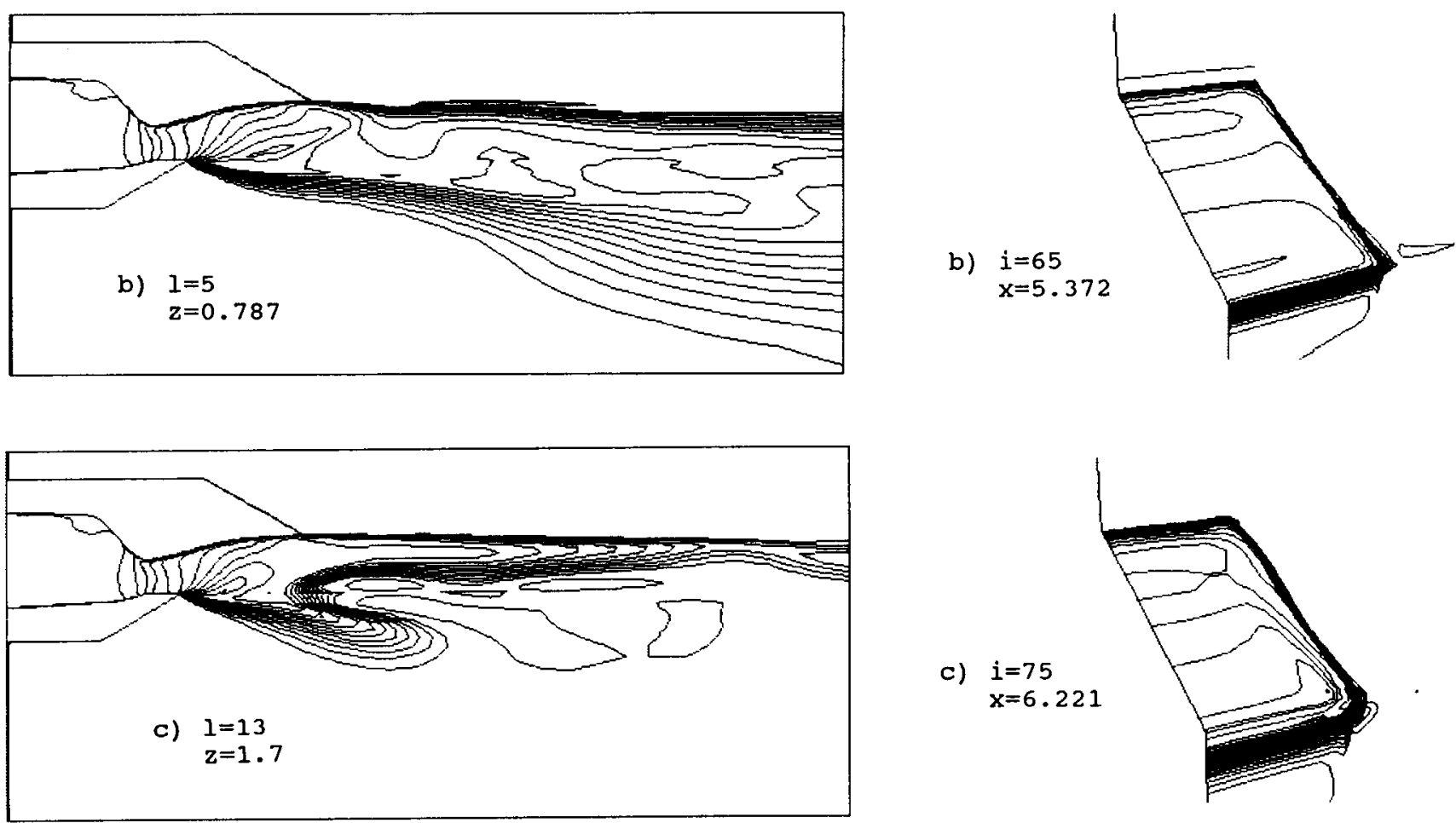

\section{c) $i=75$}

$x=6.221$
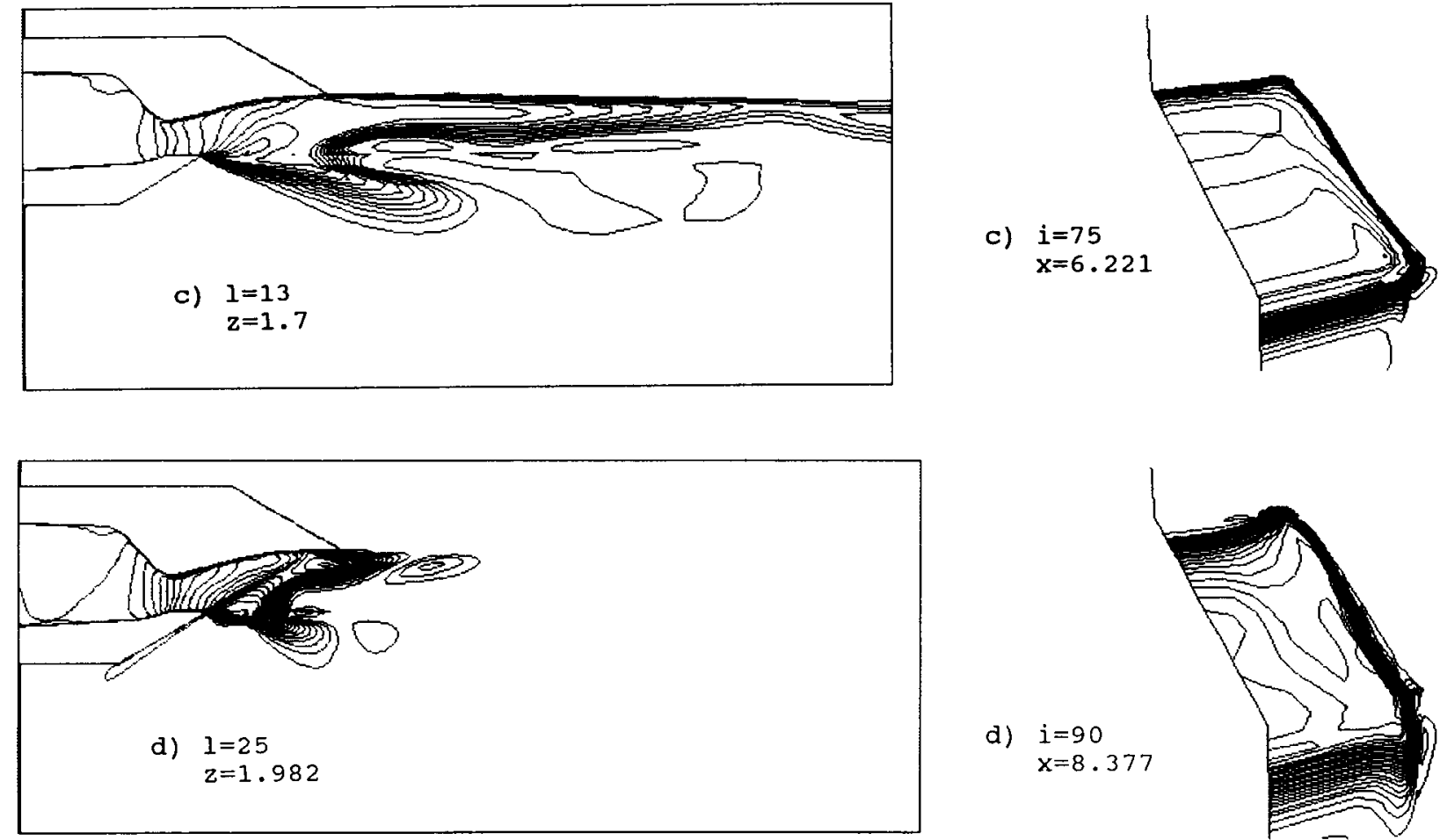

d) $i=90$

$x=8.377$

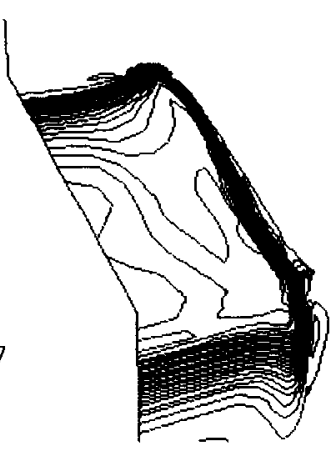

Fig. 12 Streamwise Mach Number Contours, Case 2. 


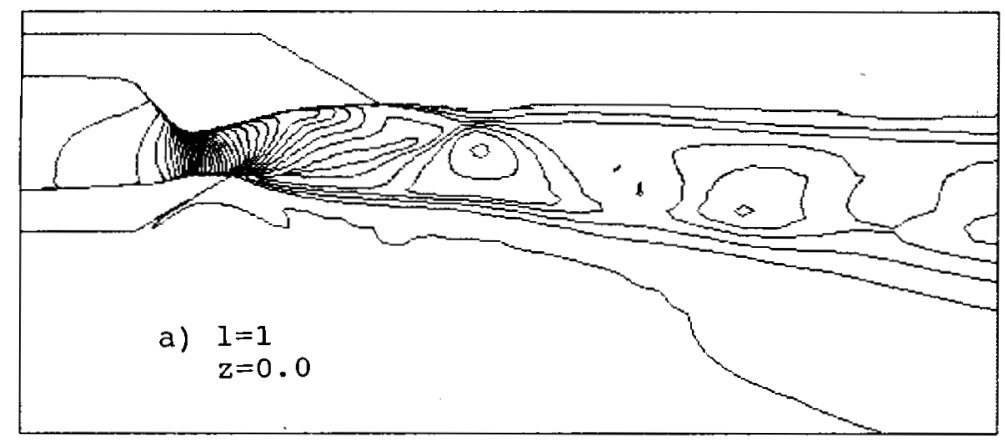

a) $i=85$

$x=7.513$
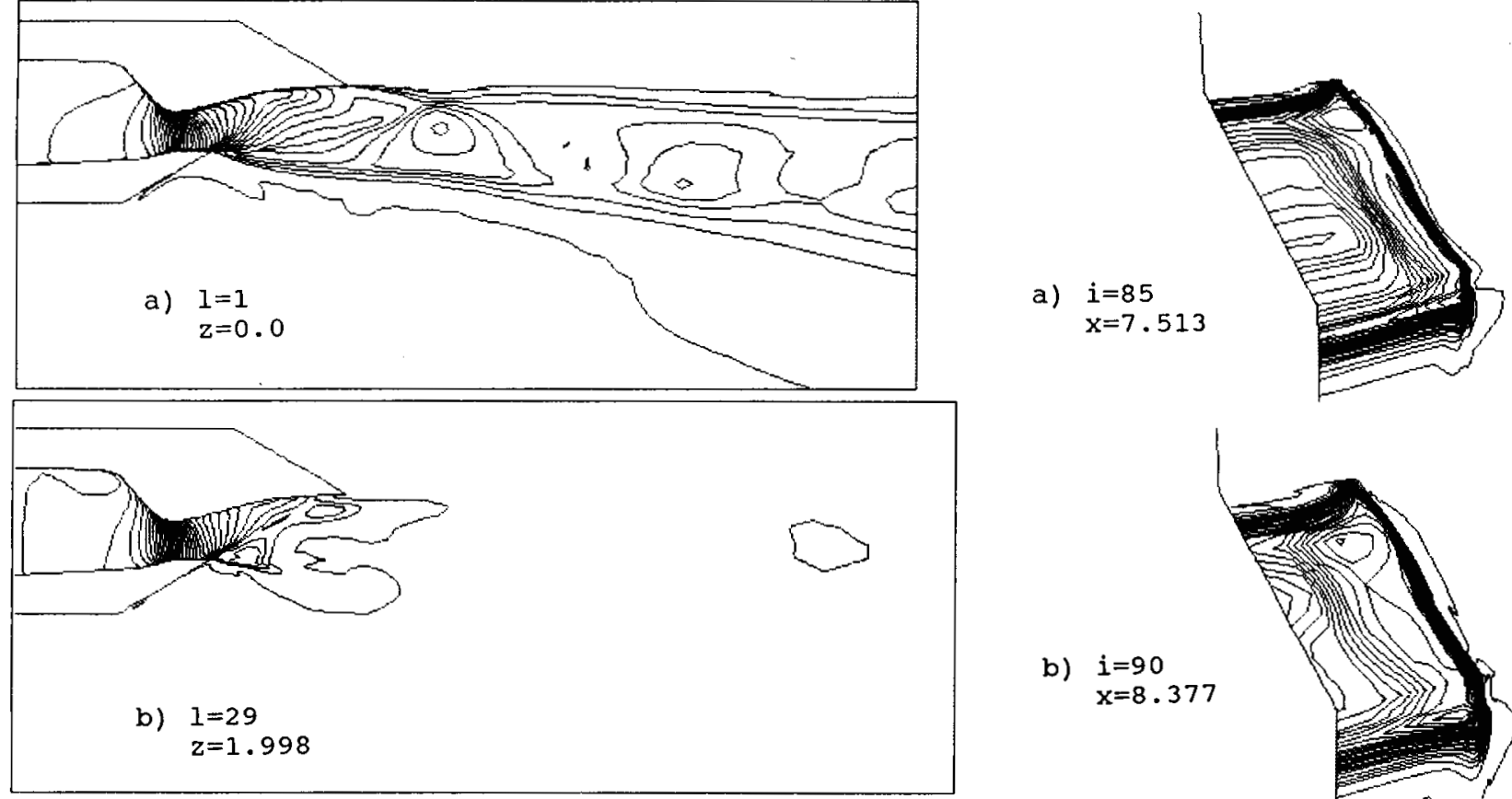

b) $i=90$

$x=8.377$

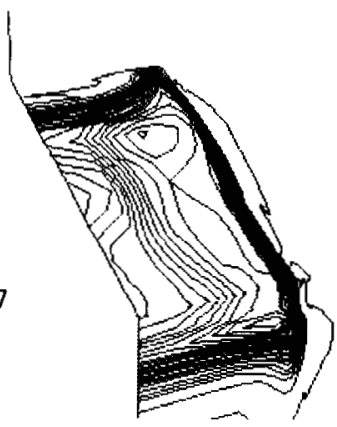

Fig. 14 Streamwise Density Contours, Case 2 .

Fig. 16 Spanwise Density Contours, Case 2 .

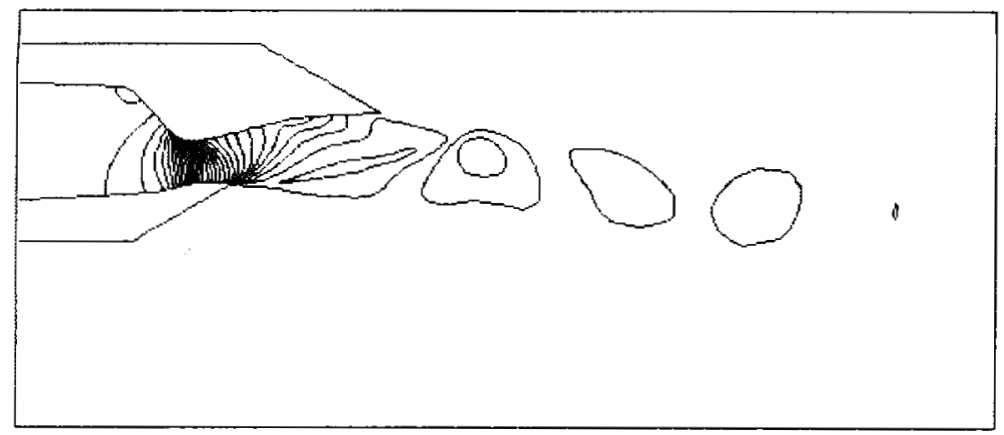

Fig. 15 Streamwise Pressure contours,

Case $2,1=1, z=0.0$.
.

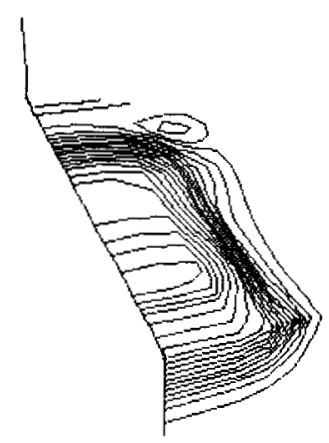

Fig. 17 Spanwise Pressure contours,

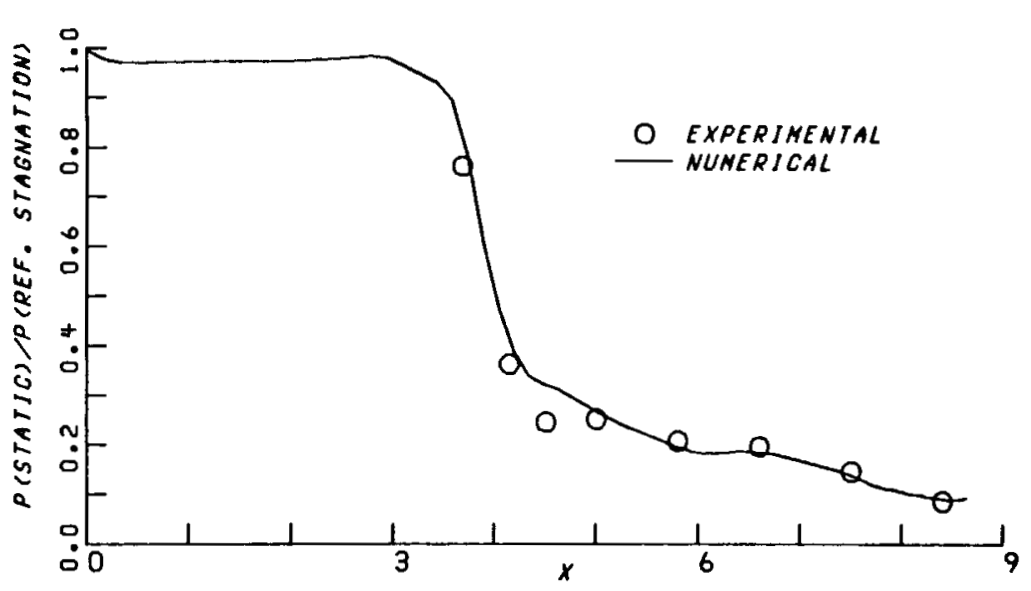

a)

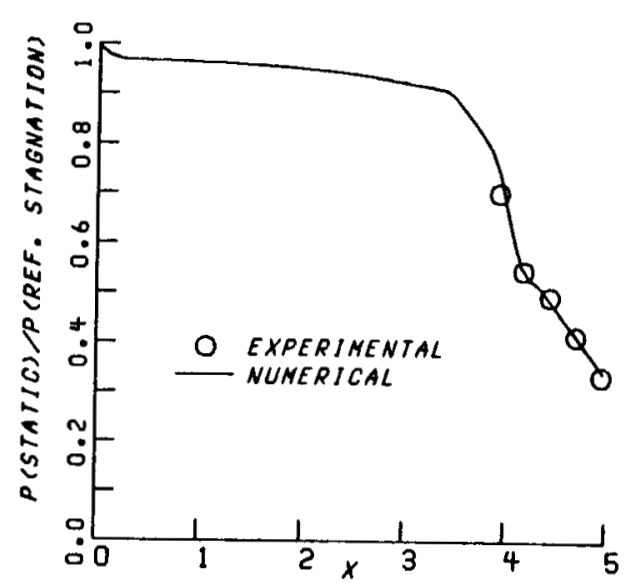

b)

Fig. 18 Wall Pressure Distributions, Case 2,

a) Upper Wall.

b) Lower Wall. 


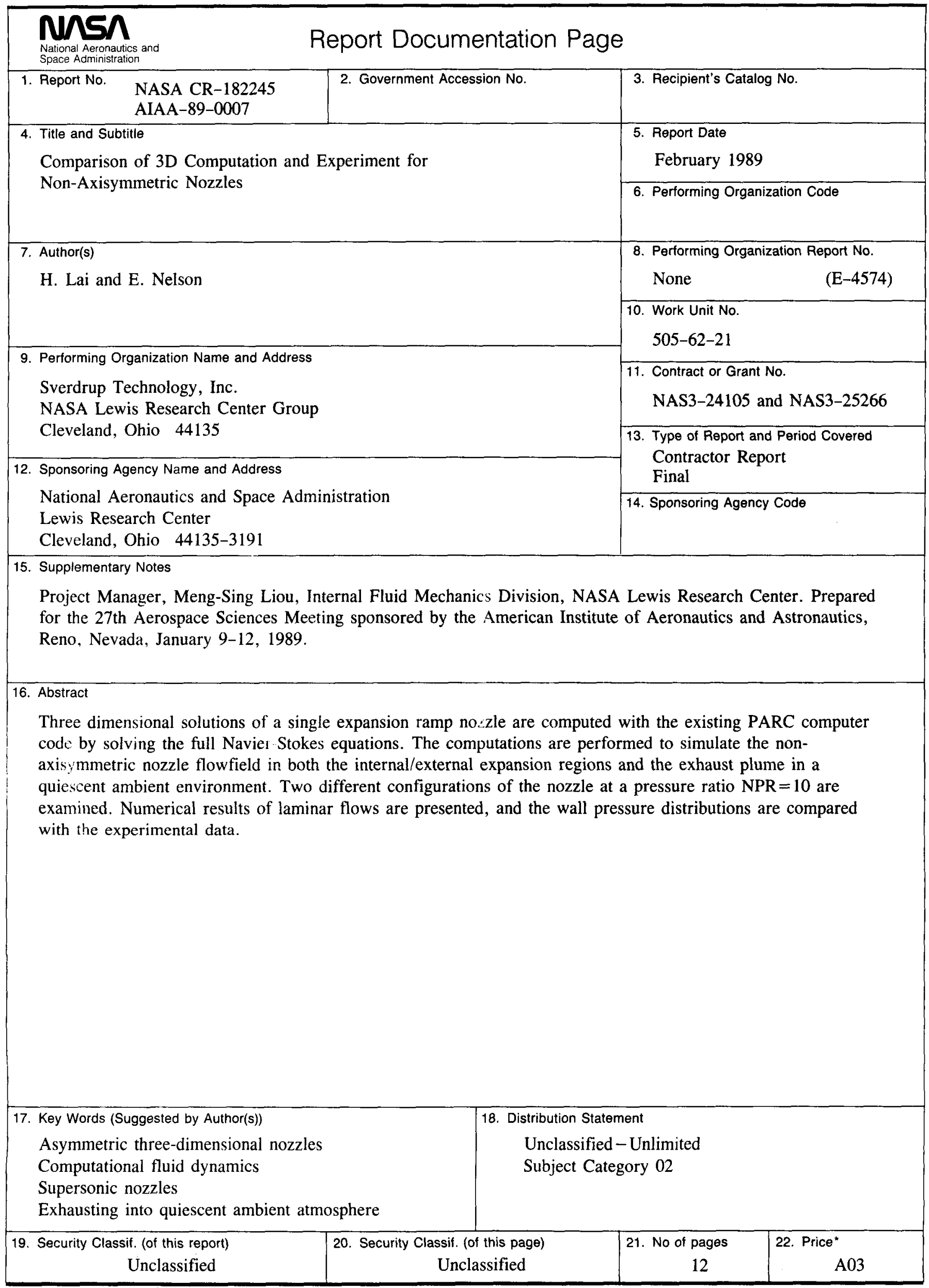

